\title{
On the Renewal Risk Model with Constant Interest Force
}

\author{
K.K. Thampi \\ Department of Statistics, SNMC, M.G.University, Kerala-683516, INDIA. \\ Email : thampisnm@yahoo.co.in \\ M.J. Jacob \\ Department of Mathematics, NITC, Calicut-673601, INDIA. \\ Email :mjj@nitc.ac.in \\ Received 24 December 2012 \\ Accepted 11 June 2013
}

\begin{abstract}
In this paper, we consider a renewal risk model with constant interest force for an insurance portfolio. We discuss equations for the survival probability and its Laplace-Stieltjes transforms have been obtained. We provide recursive algorithm for the upper and lower bounds for the ruin/survival probability under interest force. Finally, we derive an exponential integral equation for the survival probability. Some special cases are also discussed.
\end{abstract}

Keywords: Constant interest force, Generalized Exponential distribution, Laplace-Stieltjes transform, Probability of Ruin, Recursive calculation.

\section{Introduction}

The classical compound Poisson surplus process, it is often assumed that the surplus receives no interest over time. But the large portion of the surplus of the insurance companies comes from investment income. The impact of investment risk on the ruin probability and other issues of both theoretical interest and practical importance. In risk theory, there is considerable interest in the study of investment income. In this paper we use results given by Sundt and Tuegels $(1995,1997)$ and the ideas given in Jingmin et.al (2011) and Cai and Dickson (2002) to study the ruin problems for the Generalized Exponential distribution. We aim to find the bounds for the ultimate ruin probability by recursive technique. Yang (1999) considered a discrete time risk model with a constant interest force and a non-exponential upper bounds for ruin probabilities were obtained. Paulsen and Gjessing (1997) considered a diffusion perturbed classical model, a Lundberg type inequality was obtained by assuming a stochastic investment income.
Recently there has been considerable interest in extending results from classical risk theory, to more flexible general models. A general model involves the assumption that the inter-claim times are independent and identically distributed, but not necessarily exponential. The resulting surplus process is referred to as a renewal risk process or so called Sparre Andersen model proposed by Sparre Andersen (1957). Analysis of Sparre Andersen process is more difficult than the classical model, but remarkable progress has been made . One of such class of distribution for the interclaim times is Erlang distribution. Various aspects of ruin in Erlangian risk models are studied in Dickson and Hipp (1998, 2001), Cheng and Tang (2003), Sun and Yang (2004) and Li and Garrido (2004a). Thampi et al.(2007) have considered another renewal risk process in which claims occur as Generalized exponential distribution. The most related reference on the ruin probability for renewal risk model corresponding to our case is Dong and Wang (2006) which delivered integrodifferential equation for the survival and ruin probabilities with negative risk sums. They obtained 
exact expression and upper and lower bounds for the ruin probability.

There exist a vast literature on the classical risk model with constant interest force. Compared to this there are a remarkably few papers on renewal risk model under interest force. One obvious reason is that the theory is much more complicated in the renewal risk model. We mention that there are papers which are devoted to the ruin probability of renewal risk model with interest force. We do not plan to cite here a complete list of references. Recently, Konstantinides et al. (2010) considered the ruin probability $\psi_{\delta}(\mathrm{u})$ with the constant interest force $\delta>0$. They established an asymptotic expression for the ultimate ruin probability. Here we apply the methodology in Sundt and Tuegels (1995), Cai and Dickson (2002) and Dong and Wang (2006) to derive bounds for ruin/survival probabilities for a particular class of renewal risk model under interest force. The purpose of this paper is to derive some explicit expression for ruin/survival probabilities for a particular class of renewal risk process under interest force. We assume that the claims inter-arrival times have Generalized exponential distribution. We show that techniques that can be applied to produce explicit results for ruin probabilities in classical risk model under interest force can also be applied when interclaim times is Generalized exponential. We have obtained an integral equation satisfied by the ruin/survival probability under interest force. Also we derive a couple alternative expressions, an exponential integral equation for the survival probability and the other a second order differential equation satisfied by the Laplace-Stieltjes transform of the non-ruin probability.

The outline of the paper is summarized as follows. In section 2, we set out the mathematical preliminaries that help to describe the model in subsequent sections. In section 3, we apply the technique developed in Sundt and Tuegel (1995) to derive a differential equation for the survival probability in the special case when $\alpha=2$. In section 4 , we obtain bounds for the ruin probability by recurssive technique and find that the derivation is some what complicated in general, but less so when we assume $\alpha=2$. An exponential integral equation for the survival probability is derived in section 5 .

The generalized exponential distribution has been introduced by Gupta and Kundu (1999). A random variable $\mathrm{X}$ has the generalized exponential distribution with parameters $\alpha$ and $\lambda$ if it has distribution function

$$
\mathrm{F}(\mathrm{x} ; \alpha, \lambda)=\left(1-\mathrm{e}^{-\lambda \mathrm{x}}\right)^{\alpha}, \mathrm{x}>0, \alpha>0, \lambda>0 .
$$

with corresponding density function

$$
\mathrm{f}(\mathrm{x} ; \alpha, \lambda)=\alpha \lambda\left(1-\mathrm{e}^{-\lambda \mathrm{x}}\right)^{\alpha-1} \mathrm{e}^{-\lambda \mathrm{x}}, \mathrm{x}>0, \alpha>0, \lambda>0 .
$$

The generalized exponential distribution is denoted by $\operatorname{GE}(\alpha, \lambda)$. The GE distribution has many nice properties and it can be used as an alternative to Gamma and Weibull distributions in many situations(Gupta and Kundu(2001))

\section{Preliminaries}

We consider a risk process in which claim occur as a renewal process. Let $\left\{\mathrm{T}_{\mathrm{i}}\right\}_{\mathrm{i}=1}^{\infty}$ be a sequence of independent and identically distributed random variables, where $T_{i}$ denotes the time until the first claim and, for $\mathrm{i}>1$, denotes the the time between the $(\mathrm{i}-1)^{\text {th }}$ and $i^{\text {th }}$ claim. We assume that $T_{i}$ has a $\operatorname{GE}(n, \lambda)$ distribution with density function

$$
g(t)=n \lambda\left(1-e^{-\lambda t}\right)^{n-1} e^{-\lambda t}, t>0, \lambda>0
$$

where $\mathrm{n}$ is a positive integer, and distribution function

$$
\mathrm{G}(\mathrm{t})=\left(1-\mathrm{e}^{-\lambda \mathrm{t}}\right)^{\mathrm{n}}, \mathrm{t}>0, \quad \lambda>0 .
$$

For most of this paper, we illustrate ideas by restricting our attention to the case in which $n=2$. Of course, when $\mathrm{n}=1$, we have the classical risk model.

Let $\mathrm{U}_{\delta}(\mathrm{t})$ denotes the value of the reserve at time $t$. $\mathrm{U}_{\delta}(\mathrm{t})$ is governed by

$$
d U_{\delta}(t)=c d t+U_{\delta}(t) \delta d t-d X(t),
$$

that is

$$
\mathrm{U}_{\delta}(\mathrm{t})=\mathrm{ue} \mathrm{e}^{\delta \mathrm{t}}+\mathrm{cs}_{\mathrm{t}}^{-(\delta)}-\int_{0}^{\mathrm{t}} \mathrm{e}^{\delta(\mathrm{t}-\mathrm{v})} \mathrm{dX}(\mathrm{v})
$$

where $\mathrm{u}=\mathrm{U}_{\delta}(0)$ and $\mathrm{c}$ is a premium that insurance company receives per unit time. In addition to the premium income, the insurance company also receives interest of its reserves with constant force $\delta$ and $\mathrm{X}(\mathrm{t})$ denotes the accumulated amount of claims occurring in the time interval $(0, \mathrm{t}]$, that is

$$
X(t)=\sum_{j=1}^{N(t)} X_{j}
$$

We assume that $\{\mathrm{X}(\mathrm{t})\}_{\mathrm{t} \geq 0}$ is comprised of renewal claim number process $\{\mathrm{N}(\mathrm{t})\}_{\mathrm{t} \geq 0}$ whose inter-claim times $\left\{\mathrm{T}_{1}, \mathrm{~T}_{2} \ldots\right\}$ have $\operatorname{GE}(2, \lambda)$ distribution. The individual claim amounts $\mathrm{X}_{1}, \mathrm{X}_{2} \ldots$ independent of $\{\mathrm{N}(\mathrm{t})\}_{\mathrm{t} \geq 0}$ are positive, independent and identically distributed random variables with common cumulative 
distribution function $\mathrm{F}(\mathrm{x})=\mathrm{P}\{\mathrm{X} \leq \mathrm{x}\}$ and mean $\mathrm{m}=\mathrm{E}(\mathrm{X})$.

Finally,

$$
\stackrel{\mathrm{s}}{\mathrm{t}}_{\overrightarrow{-}(\delta)}=\int_{0}^{\mathrm{t}} \mathrm{e}^{\delta \mathrm{v}} \mathrm{dv}= \begin{cases}\mathrm{t} & \text { if } \delta=0 \\ \frac{\mathrm{e}^{\delta \mathrm{t}}-1}{\delta} & \text { if } \delta>0\end{cases}
$$

For convenience we will drop the index $\delta$, when the force of interest is zero. Then (1) is reduced to the usual risk process

$$
\mathrm{U}(\mathrm{t})=\mathrm{u}+\mathrm{ct}-\mathrm{X}(\mathrm{t}) \text {. }
$$

Now we define the time of ruin by

$$
\tau_{\delta}=\inf \left\{\mathrm{t}: \mathrm{U}_{\delta}(\mathrm{t})<0\right\} .
$$

Let $\psi_{\delta}(\mathrm{u})$ denote the probability that the company is ruined at sometime starting with initial reserve $u$. So

$\psi_{\delta}(\mathrm{u})=\mathrm{P}\left\{\underset{\mathrm{t} \geq 0}{\mathrm{U}} \mathrm{U}_{\delta}(\mathrm{t})<0\right\}=\mathrm{P}\left\{\tau_{\delta}<\infty \mid \mathrm{U}_{\delta}(0)=\mathrm{u}\right\}$.

We denote $\varphi_{\delta}(\mathrm{u})=1-\psi_{\delta}(\mathrm{u})$, the survival probability, that is, the probability that ruin never occurs.

Consider the risk reserve process $\mathrm{U}(\mathrm{t})=\mathrm{u}+\mathrm{ct}-\mathrm{X}(\mathrm{t})$. Let $\mathrm{Y}_{\mathrm{i}}=\mathrm{cT}_{\mathrm{i}}-\mathrm{X}_{\mathrm{i}}, \mathrm{i}=1,2, \ldots$ and $\mathrm{m}_{\mathrm{y}}(\mathrm{s})=\mathrm{E}\left\{\mathrm{e}^{\mathrm{sY}}\right\}$ be the moment generating function of $Y_{i}$. Consider the equation

$$
\mathrm{m}_{\mathrm{y}}(\mathrm{s})=\mathrm{E}\left\{\mathrm{e}^{\mathrm{sct}}\right\} \mathrm{E}\left\{\mathrm{e}^{-\mathrm{s} \mathrm{X}_{\mathrm{i}}}\right\}=1 .
$$

Clearly $\mathrm{m}_{\mathrm{y}}(0)=1$. This equation may have a second root. If such a solutions to (3), $\mathrm{s}=0$ exist, then it is unique and positive. This equation is the defining equation for the adjustment coefficient.

\section{Integral Equation for Survival Probability}

In this section, we derive an integral equation for $\varphi_{\delta}(\mathrm{u})$. Suppose that the first claim occurs at time $T_{1}=t$ and the amount of claim is $\mathrm{X}_{1}=\mathrm{x}$, the surplus just after the payment of first claim is $\mathrm{ue}^{\delta \mathrm{t}}+\mathrm{cs}_{\mathrm{t}}^{-(\delta)}-\mathrm{x}$. By considering the time and amount of first claim, the conditional probability that the company will survive is $\varphi_{\delta}\left(u e^{\delta t}+\operatorname{cs}_{t}^{-(\delta)}-x\right)$. Thus we have

that is $\varphi_{\delta}(\mathrm{u})=\int_{0}^{\infty} \mathrm{g}(\mathrm{t}) \int_{0}^{\mathrm{e}^{\delta \mathrm{t}+\mathrm{cs}^{-(\delta)}} \mathrm{t} \mid} \varphi_{\delta}\left(\mathrm{ue}^{\delta \mathrm{t}}+\mathrm{cs}_{\mathrm{t}}^{-(\delta)}-\mathrm{x}\right) \mathrm{dF}(\mathrm{x}) \mathrm{dt}$.

The change of variable $\mathrm{s}=\mathrm{e}^{\delta \mathrm{t}}+\mathrm{cs}_{\mathrm{t}}^{-(\delta)}$, we obtain $\varphi_{\delta}(\mathrm{u})=$

$2 \lambda \int_{u}^{\infty}\left((\mathrm{c}+\delta \mathrm{u})^{\lambda / \delta}(\mathrm{c}+\delta \mathrm{s})^{-1-\lambda \delta}-(\mathrm{c}+\delta \mathrm{u})^{2 \lambda / \delta}(\mathrm{c}+\delta \mathrm{s})^{-1-2 \lambda / \delta}\right) \times$

$\int_{0}^{u} \varphi_{\delta}(s-x) d F(x) d s$.

Differentiating expression (1) with respect $\mathrm{u}$ gives $(\mathrm{c}+\delta \mathrm{u}) \varphi_{\delta}^{\prime}(\mathrm{u})=$

$2 \lambda \int_{u}^{\infty}\left((\mathrm{c}+\delta \mathrm{u})^{\lambda / \delta}(\mathrm{c}+\delta \mathrm{s})^{-1-\lambda / \delta} \lambda-2 \lambda(\mathrm{c}+\delta \mathrm{u})^{2 \lambda \delta \delta}(\mathrm{c}+\delta \mathrm{s})^{-1-2 \lambda / \delta}\right) \times$

$\int_{0}^{s} \varphi_{\delta}(s-x) d F(x) d s$.

Differentiating expression (5) again with respect to $\mathrm{u}$ and rearrange the terms

$\varphi_{\delta}^{\prime \prime}(\mathrm{u})=$

$\frac{2 \lambda}{(c+\delta u)^{2}} \int_{u}^{\infty}\left[(c+\delta u)^{\lambda / \delta}(c+\delta s)^{-1-\lambda / \delta} \lambda\left(\frac{\lambda}{\delta}-1\right)-2 \delta(c+\delta u)^{2 \lambda / \delta}\right.$

$\left.(\mathrm{c}+\delta \mathrm{s})^{-1-2 \lambda / \delta}\left(\frac{2 \lambda}{\delta}-1\right)\right] \int_{0}^{\mathrm{s}} \varphi_{\delta}(\mathrm{s}-\mathrm{x}) \mathrm{dF}(\mathrm{x}) \mathrm{ds}-\frac{\lambda}{(\mathrm{c}+\delta \mathrm{u})^{2}}$

$\int_{0}^{\mathrm{u}} \varphi_{\delta}(\mathrm{u}-\mathrm{x}) \mathrm{dF}(\mathrm{x})$.

Inserting $\varphi_{\delta}^{\prime}(\mathrm{u})$ and $\varphi_{\delta}(\mathrm{u})$ into (6) and making a couple of simplification we arrive at the integrodifferential equation

$(\mathrm{c}+\delta \mathrm{u})^{2} \varphi_{\delta}^{\prime \prime}(\mathrm{u})=$

$(\mathrm{c}+\delta \mathrm{u})(3 \lambda-\delta) \varphi_{\delta}^{\prime}(\mathrm{u})-2 \lambda^{2} \varphi_{\delta}(\mathrm{u})+2 \lambda^{2} \int_{0}^{\mathrm{u}} \varphi_{\delta}(\mathrm{u}-\mathrm{x}) \mathrm{dF}(\mathrm{x})$.

Integrating both sides of (7) with respect $u$ and after simplifications

$$
\begin{aligned}
& (\mathrm{c}+\delta \mathrm{u})^{2} \varphi_{\delta}^{\prime}(\mathrm{u})= \\
& \left(\delta \mathrm{c}+\delta^{2} \mathrm{u}+3 \lambda \delta \mathrm{u}+3 \lambda \mathrm{c}\right) \varphi_{\delta}(\mathrm{u})+\mathrm{c}^{2} \varphi_{\delta}^{\prime}(0)-(3 \lambda \mathrm{c}+\delta \mathrm{c}) \varphi_{\delta}(0)- \\
& \left(3 \lambda \delta+\delta^{2}\right) \int_{0}^{\mathrm{u}} \varphi_{\delta}(\mathrm{v}) \mathrm{dv}-2 \lambda^{2} \int_{0}^{\mathrm{u}} \varphi_{\delta}(\mathrm{u}-\mathrm{x})(1-\mathrm{F}(\mathrm{x})) \mathrm{dx},
\end{aligned}
$$

which we rewrite as

$(\mathrm{c}+\delta \mathrm{u})^{2} \varphi_{\delta}^{\prime}(\mathrm{u})-\left(\delta^{2}+3 \lambda \delta\right) \mathrm{u} \varphi_{\delta}(\mathrm{u})+\left(3 \lambda \delta+\delta^{2}\right) \int_{0}^{\mathrm{u}} \varphi_{\delta}(\mathrm{v}) \mathrm{dv}$ 


$$
\begin{aligned}
= & (\delta \mathrm{c}+3 \lambda \mathrm{c}) \varphi_{\delta}(\mathrm{u})+\mathrm{c}^{2} \varphi_{\delta}^{\prime}(0)-(3 \lambda \mathrm{c}+\delta \mathrm{c}) \varphi_{\delta}(0) \\
& -2 \lambda^{2} \int_{0}^{\mathrm{u}} \varphi_{\delta}(\mathrm{u}-\mathrm{x})(1-\mathrm{F}(\mathrm{x})) \mathrm{dx} .
\end{aligned}
$$

As limit $\mathrm{u} \rightarrow \infty$, the left hand side of the expression (9) tend to a constant

$$
\mathrm{B}(\delta)=\delta(\delta+3 \lambda) \int_{0}^{\infty} \psi_{\delta}(\mathrm{u}) \mathrm{du},
$$

and the right hand side becomes

$$
(\delta c+3 \lambda c)\left(1-\varphi_{\delta}(0)\right)+c^{2} \varphi_{\delta}^{\prime}(0)-2 \lambda^{2} m .
$$

It is easily seen that $\mathrm{B}(0)=0$, we obtain

$$
\varphi_{\delta}^{\prime}(0)=\frac{\mathrm{B}(\delta)-(\delta \mathrm{c}+3 \lambda \mathrm{c})\left(1-\varphi_{\delta}(0)\right)+2 \lambda^{2} \mathrm{~m}}{\mathrm{c}^{2}} .
$$

Inserting (10) into (8), we get

$(\mathrm{c}+\delta \mathrm{u})^{2} \varphi_{\delta}^{\prime}(\mathrm{u})=$

$\left(\delta \mathrm{c}+\delta^{2} \mathrm{u}+3 \lambda \delta \mathrm{u}+3 \lambda \mathrm{c}\right) \varphi_{\delta}(\mathrm{u})+2 \lambda^{2} \mathrm{~m}+\mathrm{B}(\delta)-(\delta \mathrm{c}+3 \lambda \mathrm{c})$

$-\left(3 \lambda \delta+\delta^{2}\right) \int_{0}^{\mathrm{u}} \varphi_{\delta}(\mathrm{v}) \mathrm{dv}-2 \lambda^{2} \int_{0}^{\mathrm{u}} \varphi_{\delta}(\mathrm{u}-\mathrm{x})(1-\mathrm{F}(\mathrm{x})) \mathrm{dx}$.

Integrating this expression again with respect to $\mathrm{u}$ gives

$$
\begin{aligned}
& \varphi_{\delta}(\mathrm{u})=\frac{1}{(\mathrm{c}+\delta \mathrm{u})^{2}}\left[(3 \delta \mathrm{c}+3 \lambda \mathrm{c}) \int_{0}^{\mathrm{u}} \varphi_{\delta}(\mathrm{v}) \mathrm{dv}+\left(6 \lambda \delta+4 \delta^{2}\right)\right. \\
& \int_{0}^{\mathrm{u}} \mathrm{v} \varphi_{\delta}(\mathrm{v}) \mathrm{dv}-\left(3 \lambda \delta+\delta^{2}\right) \mathrm{u} \int_{0}^{\mathrm{u}} \varphi_{\delta}(\mathrm{v}) \mathrm{dv}+\mathrm{c}^{2} \varphi_{\delta}(0)+ \\
& \left.\left(\mathrm{B}(\delta)-(\delta \mathrm{c}+3 \lambda \mathrm{c})+2 \lambda^{2} \mathrm{~m}\right) \mathrm{u}-2 \lambda^{2} \int_{0}^{\mathrm{u}} \int_{0}^{\mathrm{v}} \varphi_{\delta}(\mathrm{v}-\mathrm{x})(1-\mathrm{F}(\mathrm{x})) \mathrm{dxdv}\right]
\end{aligned}
$$

When $\delta=0$, (12) reduces to

$$
\begin{aligned}
& c^{2} \varphi(u)= \\
& 3 \lambda c \int_{0}^{u} \varphi(v) d v+c^{2} \varphi(0)+\left(2 \lambda^{2} m-3 \lambda c\right) u-2 \lambda^{2} m \int_{0}^{u} \varphi(u-x) F_{1}(x) d x,
\end{aligned}
$$

with the integrated tail distribution of $\mathrm{F}$ is given by

$$
\mathrm{F}_{1}(\mathrm{x})=\frac{1}{\mathrm{~m}} \int_{0}^{\mathrm{x}}[1-\mathrm{F}(\mathrm{y})] \mathrm{dy} \text {. }
$$

and $c^{2} \varphi(0)=\frac{3 \lambda c-2 \lambda^{2} m}{r}$ where $r$ is the positive solution of the equation (3).

\subsection{Laplace Transform}

The appearance of convolution in (13) suggest that it is better to use Laplace transform to get an explicit expression for non-ruin probability when the interest force is zero. So we introduce

$$
\hat{\varphi}(\mathrm{s})=\int_{0}^{\infty} \mathrm{e}^{-\mathrm{sv}} \varphi(\mathrm{v}) \mathrm{dv},
$$

and

$$
\hat{f}(s)=\int_{0}^{\infty} e^{-s y} d F(y) .
$$

Taking the Laplace transform of the integral equation (13), we obtain

$$
\hat{\varphi}(s)=\frac{c^{2} s \varphi(0)+2 \lambda^{2} m-3 \lambda c}{c^{2} s^{2}-3 \lambda c s+2 \lambda^{2}(1-\hat{f}(s))}
$$

Such expressions can be easily inverted using mathematical software packages.

\section{Recursive Calculation of Ruin Probabilities}

In this section, we derive bounds for the ruin probability in renewal risk model by recursive techniques. Exact solutions for the ruin probability $\psi_{\delta}(\mathrm{u})$ are difficult to find. We find the bounds for $\varphi_{\delta}(\mathrm{u})$ by discretizing the integral equation (12). For any $\mathrm{h}>0$ and $\mathrm{k}=1,2, \ldots$ we have

$$
\varphi_{\delta}^{\left(\mathrm{h}^{-}\right)}(\mathrm{hk}) \leq \varphi_{\delta}(\mathrm{hk}) \leq \varphi_{\delta}^{\left(\mathrm{h}^{+}\right)}(\mathrm{hk}),
$$

with

$$
\begin{aligned}
& \varphi_{\delta}^{\left(\mathrm{h}^{+}\right)}(\mathrm{hk})=\frac{1}{(\mathrm{c}+\delta \mathrm{kh})^{2}-\mathrm{p}_{1} \mathrm{~h}-\mathrm{p}_{3}(2 \mathrm{k}-1) \frac{\mathrm{h}^{2}}{2}} \\
& \left\{\mathrm{p}_{1} \sum_{\mathrm{j}=1}^{\mathrm{k}-1} \varphi_{\delta}^{\left(\mathrm{h}^{+}\right)}(\mathrm{jh}) \mathrm{h}-\mathrm{p}_{2} \mathrm{kh} \sum_{\mathrm{j}=0}^{\mathrm{k}-1} \varphi_{\delta}^{\left(\mathrm{h}^{-}\right)}(\mathrm{jh}) \mathrm{h}+\right. \\
& \mathrm{p}_{3} \sum_{\mathrm{j}=1}^{\mathrm{k}-1}(2 \mathrm{j}-1) \frac{\mathrm{h}^{2}}{2} \varphi_{\delta}^{\left(\mathrm{h}^{+}\right)}(\mathrm{jh})+\mathrm{c}^{2} \varphi_{\delta}(0)+\mathrm{p}_{4} \mathrm{kh}- \\
& \left.\mathrm{p}_{5} \sum_{\mathrm{j}=1}^{\mathrm{k}} \varphi_{\delta}^{\left(\mathrm{h}^{-}\right)}((\mathrm{k}-\mathrm{j}) \mathrm{h}) \mathrm{f}_{\mathrm{j}}(\mathrm{h})\right\}
\end{aligned}
$$

$$
\begin{aligned}
& \varphi_{\delta}^{\left(\mathrm{h}^{-}\right)}(\mathrm{hk})=\frac{1}{(\mathrm{c}+\delta \mathrm{kh})^{2}+\mathrm{p}_{2} \mathrm{kh}^{2}+\mathrm{p}_{5} \mathrm{f}_{1}(\mathrm{~h})} \\
& \left\{\mathrm{p}_{1} \sum_{\mathrm{j}=0}^{\mathrm{k}-1} \varphi_{\delta}^{\left(\mathrm{h}^{-}\right)}(\mathrm{jh}) \mathrm{h}-\mathrm{p}_{2} \mathrm{kh} \sum_{\mathrm{j}=1}^{\mathrm{k}-1} \varphi_{\delta}^{\left(\mathrm{h}^{+}\right)}(\mathrm{jh}) \mathrm{h}+\right. \\
& \mathrm{p}_{3} \sum_{\mathrm{j}=1}^{\mathrm{k}}(2 \mathrm{j}-1) \frac{\mathrm{h}^{2}}{2} \varphi_{\delta}^{\left(\mathrm{h}^{-}\right)}((\mathrm{j}-1) \mathrm{h})+\mathrm{c}^{2} \varphi_{\delta}(0)+\mathrm{p}_{4} \mathrm{kh}-
\end{aligned}
$$




$$
\begin{gathered}
\left.\mathrm{p}_{5} \sum_{\mathrm{j}=1}^{\mathrm{k}-1} \varphi_{\delta}^{\left(\mathrm{h}^{+}\right)}((\mathrm{k}-\mathrm{j}) \mathrm{h}) \mathrm{f}_{\mathrm{j}+1}(\mathrm{~h})\right\}, \\
\mathrm{f}_{\mathrm{k}}(\mathrm{h})=\int_{\mathrm{h}(\mathrm{k}-1)}^{\mathrm{hk}}(1-\mathrm{F}(\mathrm{y})) \mathrm{dy} .
\end{gathered}
$$

where $\mathrm{p}_{1}=3 \delta \mathrm{c}+3 \lambda \mathrm{c}, \mathrm{p}_{2}=3 \lambda \delta+\delta^{2}, \mathrm{p}_{3}=6 \lambda \delta+4 \delta^{2}$, $\mathrm{p}_{4}=\mathrm{B}(\delta)-(\delta \mathrm{c}+3 \lambda \mathrm{c})+2 \lambda^{2} \mathrm{~m}$ and $\mathrm{p}_{5}=2 \lambda^{2} \mathrm{~m}$.

The algorithm implements lower and upper bounds successively. The two sided bounds for the survival probability is of theoretical importance, as it can hardly be used for direct numerical computations. For the interest free model, however, the algorithm give useful two sided bounds for $\varphi(u)$.

A slightly different method is used to obtain the bounds for the ruin probability in a Classical risk model with interest in which

$$
\mathrm{G}(\mathrm{t})=1-\mathrm{e}^{-\lambda \mathrm{t}}, \mathrm{t} \geq 0, \quad \lambda>0 .
$$

For compound Poisson model with interest $\delta$, there exist a unique positive solution, $\gamma$, such that

$$
\frac{1}{\mathrm{E}\left\{\mathrm{e}^{\gamma \mathrm{X}}\right\}}=\frac{\lambda}{\mathrm{c}} \int_{0}^{\infty} \frac{\mathrm{e}^{-\gamma \mathrm{x}}}{\left(1+\frac{\delta \mathrm{x}}{\mathrm{c}}\right)^{\frac{\lambda}{\delta}+1}} \mathrm{dx}
$$

The solution to equation (15) is called the adjustment coefficient for compound Poisson risk model modified by interest. The right hand side of equation (15) is simplified to

$$
\frac{\lambda}{\mathrm{c}} \int_{0}^{\infty} \frac{\mathrm{e}^{-\gamma \mathrm{y}}}{\left(1+\frac{\delta \mathrm{y}}{\mathrm{c}}\right)^{\frac{\lambda}{\delta}+1}} \mathrm{dy}=\frac{\lambda}{\mathrm{c}} \mathrm{e}^{\frac{\mathrm{c} \gamma}{\delta}} \gamma^{\frac{\lambda}{\delta}}\left(\frac{\delta}{\mathrm{c}}\right)^{-\left(\frac{\lambda}{\delta}+1\right)} \Gamma\left(-\frac{\lambda}{\delta}, \frac{\mathrm{c} \gamma}{\delta}\right)
$$

where $\Gamma(n, x)=\int_{x}^{\infty} y^{n-1} e^{-y} d y, n>0, x \geq 0$ is the incomplete Gamma function.

Theorem 4.1 Assume that the adjustment coefficient $\gamma>0$, exist, then

$$
a_{-} e^{-\gamma u} \leq \psi_{\delta}(u) \leq a_{+} e^{-\gamma u},
$$

for $u \geq 0$, where $a_{-}=\inf _{x \in\left[0, x_{0}\right)} \frac{e^{\gamma x} \int_{x}^{\infty} \bar{F}(y) d y}{\int_{x}^{\infty} e^{\gamma y} \bar{F}(y) d y}$ and $a_{+}=\sup _{x \in\left[0, x_{0}\right)} \frac{e^{\gamma x} \int_{x}^{\infty} \bar{F}(y) d y}{\int_{x}^{\infty} e^{\gamma y} \bar{F}(y) d y}$.

Proof: The proof can be given by going along the same lines of the proof of (Theorem 5.4.1, Rolski et.al) with some obvious modifications.

This is known as two sided Lundberg bounds for the ruin function $\psi_{\delta}(\mathrm{u})$. Furthermore, for all $\mathrm{u} \geq 0$

$\psi_{\delta}(\mathrm{u})<\mathrm{a}_{+} \mathrm{e}^{-\gamma \mathrm{u}}$ if $\mathrm{a}_{+}>\psi_{\delta}(0)$ and $\mathrm{a}_{-} \mathrm{e}^{-\gamma \mathrm{u}}<\psi_{\delta}(\mathrm{u})$ if $\mathrm{a}_{-}<\psi_{\delta}(0)$.

Note that,

$\mathrm{a}_{+} \geq \frac{\int_{0}^{\infty} \overline{\mathrm{F}}(\mathrm{y}) \mathrm{dy}}{\int_{0}^{\infty} \mathrm{e}^{\gamma \mathrm{y}} \overline{\mathrm{F}}(\mathrm{y}) \mathrm{dy}}=\frac{\gamma \mathrm{m}}{\mathrm{m}_{\mathrm{x}}(\gamma)-1}=\psi_{\delta}(0)$

and analogously for $\mathrm{a}_{-} \leq \psi_{\delta}(0)$.

Let $\mathrm{X}$ have an exponential distribution with $F(x)=1-e^{-\beta x}$. In this case, an explicit formula for $\psi_{\delta}(\mathrm{u})$ is also available, namely

$$
\psi_{\delta}(\mathrm{u})=\frac{\lambda \Gamma\left(\frac{\lambda}{\delta}, \beta\left(\mathrm{u}+\frac{\mathrm{c}}{\delta}\right)\right)}{\delta \Gamma\left(\frac{\lambda}{\delta}+1, \frac{\mathrm{c} \beta}{\delta}\right)} .
$$

We use Frobenius series method to derive this formula, which is considered to be the simplest method.

Numerical examples are given to illustrate the application of explicit formula and its upper bounds in compound Poisson risk model with interest. For numerical illustration, we set $\mathrm{c}=1.1, \lambda=2$ and $\beta=3$. For interest factor, we consider two different values for $\delta=0.05$ and 0.10 . The corresponding values of the adjustment coefficient $\gamma$ are 1.20879 and 1.23466 respectively. We compare the upper bounds with exact values of ruin probabilities in classical risk model under interest force in Table 1 and 2.

Table: 1 Ruin Probabilities when $\delta=0.05$

\begin{tabular}{|c|c|c|c|}
\hline $\mathrm{u}$ & Exact Ruin & Upper Bounds & Ruin $(\delta=0)$ \\
\hline 0 & 0.586092 & 0.586092 & 0.606061 \\
\hline 1 & 0.156639 & 0.174982 & 0.185891 \\
\hline 2 & 0.039133 & 0.052242 & 0.057017 \\
\hline 3 & 0.009184 & 0.015597 & 0.017488 \\
\hline 4 & 0.002034 & 0.004657 & 0.005364 \\
\hline 5 & 0.000427 & 0.001390 & 0.001645 \\
\hline
\end{tabular}


Table: 2 Ruin Probabilities when $\delta=0.10$

\begin{tabular}{|c|c|c|c|}
\hline $\mathrm{u}$ & Exact Ruin & Upper Bounds & Ruin $(\delta=0)$ \\
\hline 0 & 0.570308 & 0.570308 & 0.606061 \\
\hline 1 & 0.136032 & 0.165922 & 0.185891 \\
\hline 2 & 0.028915 & 0.048272 & 0.057017 \\
\hline 3 & 0.005560 & 0.014044 & 0.017488 \\
\hline 4 & 0.000979 & 0.004086 & 0.005364 \\
\hline 5 & 0.000160 & 0.001189 & 0.001645 \\
\hline
\end{tabular}

\section{Alternative Expressions for $\varphi_{\delta}(\mathrm{u})$}

In this section we derive a couple of alternative expressions for the non-ruin probability.

After simplifications and rearrangement on (11), we obtain the equation

$\varphi_{\delta}^{\prime}(\mathrm{u})-\frac{3 \lambda+\delta}{(\mathrm{c}+\delta \mathrm{u})} \varphi_{\delta}(\mathrm{u})=\frac{2 \lambda^{2} \mathrm{~m}+\mathrm{B}(\delta)-(\delta \mathrm{c}+3 \lambda \mathrm{c})}{(\mathrm{c}+\delta \mathrm{u})^{2}}-$
$\frac{\left(3 \lambda \delta+\delta^{2}\right)}{(\mathrm{c}+\delta \mathrm{u})^{2}} \int_{0}^{\mathrm{u}} \varphi_{\delta}(\mathrm{v}) \mathrm{dv}-\frac{2 \lambda^{2}}{(\mathrm{c}+\delta \mathrm{u})^{2}} \int_{0}^{\mathrm{u}} \varphi_{\delta}(\mathrm{u}-\mathrm{x})(1-\mathrm{F}(\mathrm{x})) \mathrm{dx}$.

Let

$$
C(u)=e^{-\int_{0}^{u} \frac{3 \lambda+\delta}{c+\delta v} d v},
$$

which is simplified to

$$
\mathrm{C}(\mathrm{u})=\left(1+\frac{\delta \mathrm{u}}{\mathrm{c}}\right)^{-1-\frac{3 \lambda}{\delta}} \text {. }
$$

Multiplying by $\mathrm{C}(\mathrm{u})$ on both sides of (19), and integrate from 0 to $\mathrm{u}$, we get

$\left(1+\frac{\delta \mathrm{u}}{\mathrm{c}}\right)^{-1-\frac{3 \lambda}{\delta}} \varphi_{\delta}(\mathrm{u})-\varphi_{\delta}(0)=$
$\frac{1}{3 \lambda \mathrm{c}+2 \delta \mathrm{c}}\left(1+\left(1+\frac{\delta \mathrm{u}}{\mathrm{c}}\right)^{-2-\frac{3 \lambda}{\delta}}\right)\left(2 \lambda^{2} \mathrm{~m}-3 \lambda \mathrm{c}-\delta \mathrm{c}+\mathrm{B}(\delta)\right)-$

$\left(3 \lambda \delta+\delta^{2}\right) \int_{0}^{\mathrm{u}}\left(1+\frac{\delta \mathrm{v}}{\mathrm{c}}\right)^{-1-\frac{3 \lambda}{\delta}} \int_{0}^{\mathrm{v}} \varphi_{\delta}(\mathrm{y}) \mathrm{dydv}-$

$\frac{2 \lambda^{2}}{c^{2}} \int_{0}^{u}\left(1+\frac{\delta v}{c}\right)^{-3-\frac{3 \lambda}{\delta}} \int_{0}^{v} \varphi_{\delta}(v-y)(1-F(y)) d y d v$

$$
\begin{aligned}
& \mathrm{e}^{-\frac{3 \lambda}{\mathrm{c}} \mathrm{u}} \varphi(\mathrm{u})=\varphi(0)+\left(\frac{2 \lambda \mathrm{m}}{3 \mathrm{c}}-1\right)\left(1-\mathrm{e}^{-\frac{3 \lambda}{\mathrm{c}} \mathrm{u}}\right)- \\
& \left.\frac{2 \lambda^{2} \mathrm{~m}}{\mathrm{c}^{2}} \int_{0}^{\mathrm{u}}\left\{\mathrm{e}^{-\frac{3 \lambda}{\mathrm{c}} \mathrm{v}} \int_{0}^{\mathrm{v}} \varphi(\mathrm{v}-\mathrm{x}) \mathrm{dF}_{1}(\mathrm{x})\right)\right\} \mathrm{dv} .
\end{aligned}
$$

Therefore the multiplication of $\mathrm{C}(\mathrm{u})$ with $\varphi_{\delta}(\mathrm{u})$ leads to an exponentially diminishing survival probability function.

\section{Theorem:}

The Laplace-Stieltjes transform of equation $\varphi_{\delta}(\mathrm{u})$ satisfies the following second order differential equation: $\delta^{2} \mathrm{~s}^{2} \hat{\varphi}_{\delta}^{\prime \prime}(\mathrm{s})-\left(2 \delta \mathrm{cs}-3 \delta^{2}-3 \delta \lambda\right) \mathrm{s} \hat{\varphi}_{\delta}^{\prime}(\mathrm{s})+\left[\mathrm{c}^{2} \mathrm{~s}^{2}-3 \mathrm{cs}(\lambda+\delta)+\right.$
$\left.(\delta+\lambda)(\delta+2 \lambda)-2 \lambda^{2} \hat{\mathrm{f}}(\mathrm{s})\right] \hat{\varphi}_{\delta}(\mathrm{s})=\left(\mathrm{c}^{2} \mathrm{~s}-\delta \mathrm{c}-3 \lambda \mathrm{c}\right) \varphi_{\delta}(0)+\mathrm{c}^{2} \varphi_{\delta}^{\prime}(0)$,

where $\hat{\varphi}_{\delta}(\mathrm{s})=\int_{0}^{\infty} \mathrm{e}^{-\mathrm{su}} \varphi_{\delta}(\mathrm{u}) \mathrm{du}, \hat{\varphi}_{\delta}^{\prime}(\mathrm{s})=\frac{\mathrm{d}}{\mathrm{ds}} \hat{\varphi}_{\delta}(\mathrm{s})$,

$\hat{\varphi}_{\delta}^{\prime \prime}(\mathrm{s})=\frac{\mathrm{d}^{2}}{\mathrm{ds}^{2}} \hat{\varphi}_{\delta}(\mathrm{s})$ and $\varphi_{\delta}^{\prime}(0)=\left.\frac{\mathrm{d}}{\mathrm{du}} \varphi_{\delta}(\mathrm{u})\right|_{\mathrm{u}=0}$.

Proof: Consider the second order differential equation obtained in (7).

$(\mathrm{c}+\delta \mathrm{u})^{2} \varphi_{\delta}^{\prime \prime}(\mathrm{u})=$

$(\mathrm{c}+\delta \mathrm{u})(3 \lambda-\delta) \varphi_{\delta}^{\prime}(\mathrm{u})-2 \lambda^{2} \varphi_{\delta}(\mathrm{u})+2 \lambda^{2} \int_{0}^{\mathrm{u}} \varphi_{\delta}(\mathrm{u}-\mathrm{x}) \mathrm{dF}(\mathrm{x})$.

Taking Laplace transform on both sides of the equation, we get

$$
\begin{aligned}
& \mathrm{c}^{2} \mathrm{~s}^{2} \mathrm{~L}_{\mathrm{s}}\left\{\varphi_{\delta}(\mathrm{u})\right\}+2 \mathrm{c} \delta \mathrm{L}_{\mathrm{s}}\left\{\mathrm{u} \varphi_{\delta}^{\prime \prime}(\mathrm{u})\right\}+\delta^{2} \mathrm{~L}_{\mathrm{s}}\left\{\mathrm{u}^{2} \varphi_{\delta}^{\prime \prime}(\mathrm{u})\right\}- \\
& \mathrm{c}^{2} \mathrm{~s} \varphi_{\delta}(0)-\mathrm{c}^{2} \varphi_{\delta}^{\prime}(0)=3 \lambda \mathrm{cL} \mathrm{s}_{\mathrm{s}}\left\{\varphi_{\delta}^{\prime}(\mathrm{u})\right\}+3 \lambda \delta \mathrm{L}_{\mathrm{s}}\left\{\mathrm{u} \varphi_{\delta}^{\prime}(\mathrm{u})\right\}- \\
& \mathrm{c} \delta \mathrm{L}_{\mathrm{s}}\left\{\varphi_{\delta}^{\prime}(\mathrm{u})\right\}-\delta^{2} \mathrm{~L}_{\mathrm{s}}\left\{\mathrm{u} \varphi_{\delta}^{\prime}(\mathrm{u})\right\}-2 \lambda^{2} \mathrm{~L}_{\mathrm{s}}\left\{\varphi_{\delta}(\mathrm{u})\right\}+ \\
& 2 \lambda^{2} \mathrm{~L}_{\mathrm{s}}\left\{\varphi_{\delta}(\mathrm{u}-\mathrm{x}) \mathrm{f}(\mathrm{x})\right\},
\end{aligned}
$$

where $\mathrm{L}_{\mathrm{s}}\left(\varphi_{\delta}(\mathrm{u})\right)=\hat{\varphi}_{\delta}(\mathrm{s})$. Then we have the following identities

$$
\begin{aligned}
& \mathrm{L}_{\mathrm{s}}\left(\varphi_{\delta}^{\prime}(\mathrm{u})\right)=-\varphi_{\delta}(0)+\mathrm{s} \mathrm{L}_{\mathrm{s}}\left(\varphi_{\delta}(\mathrm{u})\right), \\
& \mathrm{L}_{\mathrm{s}}\left(\mathrm{u} \hat{\varphi}_{\delta}^{\prime}(\mathrm{s})\right)=-\mathrm{L}_{\mathrm{s}}\left(\varphi_{\delta}(\mathrm{u})\right)-\mathrm{s} \varphi_{\delta}^{\prime}(\mathrm{s}), \\
& \mathrm{L}_{\mathrm{s}}\left(\mathrm{u} \hat{\varphi}_{\delta}^{\prime \prime}(\mathrm{s})\right)=-\mathrm{L}_{\mathrm{s}}\left(\varphi_{\delta}^{\prime}(\mathrm{u})\right)-\mathrm{s}\left(\mathrm{L}_{\mathrm{s}}\left(\varphi_{\delta}(\mathrm{u})\right)+\mathrm{s} \varphi_{\delta}^{\prime}(\mathrm{s})\right), \\
& \mathrm{L}_{\mathrm{s}}\left(\mathrm{u}^{2} \hat{\varphi}_{\delta}^{\prime \prime}(\mathrm{s})\right)=2 \mathrm{~L}_{\mathrm{s}}\left(\varphi_{\delta}(\mathrm{u})\right)+4 \mathrm{~s} \varphi_{\delta}^{\prime}(\mathrm{s})+\mathrm{s}^{2} \hat{\varphi}_{\delta}^{\prime \prime}(\mathrm{s}) .
\end{aligned}
$$

(22) can be easily proved by inserting these identities in (23). The identities are proved by integration by parts.

Remarks: When $\delta=0$, we get the same expression as in (23). That is

$$
\hat{\varphi}(s)=\frac{\left(c^{2} s-3 \lambda c\right) \varphi(0)+c^{2} \varphi^{\prime}(0)}{c^{2} s^{2}-3 \lambda c s+2 \lambda^{2}(1-\hat{f}(s))}
$$


We can eliminate the term $\varphi^{\prime}(0)$ by considering the maximum of the aggregate loss process associated with the surplus process when $\delta=0$ and it follows that

$$
c^{2} \varphi^{\prime}(0)-3 \lambda c \varphi(0)=-3 \lambda c+2 \lambda^{2} m .
$$

In conclusion, the results in this paper give bounds for the ruin/survival probability in a Sparre Andersen risk model with constant interest force. The technique that is applied to produce explicit results for the ruin probability in the classical risk model under interest force can also be applied when inter-claim times have Generalized exponential. In section 4, a recursive algorithm is derived to obtain the the bounds for $\varphi_{\delta}(\mathrm{u})$. The algorithm yields both lower and upper bound successively. The bounds for the ruin probability in the compound Poisson model are given with numerical illustrations. A couple of alternative expressions for $\varphi_{\delta}(\mathrm{u})$ are also derived.

\section{Acknowledgements}

We would like to thank the anonymous referee for the constructive suggestions and comments on the previous versions of this paper.

\section{References}

1. Andersen, S.E. On the collective risk theory of risk in the case of contagion between the claims. Trans. of the $X V$ Intnal. congress of Acturies, 2, (1957) : 212-219.

2. Cai, J., Dickson, D.C.M. Upper bounds for ultimate ruin probabilities in the Sparre Andersen model with interest. Insurance: Mathematics and Economics, 32(1), (2003): 61-71.

3. Cheng, Y., Tang, Q Moments of surplus before ruin and deficit at ruin in the Erlang(2) risk process. North American Actuarial Journal, 7, (2003) 1-12.

4. Dickson, D.C.M., Hipp, C. Ruin probabilities for Erlang(2) risk process. Insurance: Mathematics and Economics, 22, (1998): 25-262.

5. Dickson, D.C.M., Hipp, C. On the time to ruin for Erlang(2) risk process. Insurance: Mathematics and Economics, 29, (2001): 333-344.

6. Dong, Y., Wang, G. Ruin probability for renewal risk model with negative risk sums. Journal of Industrial and Management Optimization, 2(2), (2006): 229-236.

7. Gupta, R.D., Kundu, D., Generalized Exponential distribution, Astrl \& New Zealand J. Statist., 41(2), (1999): $173-88$.

8. Gupta, R.D., Kundu, D., Generalized Exponential distribution: Different methods of estimation. J. Statist. Comput. Simul., 69, (2001) : 315 - 337.

9. Jingmin, H.E.,Rong, W.U., Jiafeng, CUI. Upper bounds for the ruin probability in a risk model with interest force whose premiums depend on the backward recurrence time process. Advances in Mathematics, 40(4), (2011) : $501-511$.
10. Konstantinides, D.G., Ng, K.W., Tang, Q. The probabilities of absolute ruin in the renewal risk model with constant force of interest, Journal of Applied Probability, 47(2), (2010): 323-334.

11. Li, S. and Garrido, J. On ruin for the Erlang(n) risk process. Insurance: Mathematics and Economics, 31, (2004a): 391-408.

12. Paulson. J and Gjessing. H.K. Optimal choice of dividend barriers for a risk process with stochastic pattern on investments, Insurance: Mathematics and Economics, 20, (1997): 215-223.

13. Rolski, T., Schmidli, H., Schmidt, V., Tuegels, J. Stochastic Processes for Insurance and Finance. John Wiley \& Sons (1999)

14. Sun, L. and Yang, H. On the joint distribution of surplus immediately before ruin and deficit at ruin for $\operatorname{Erlang}(2)$ risk processes. Insurance: Mathematics and Economics, 34, (2004): 121 - 125.

15. Sundt, S., and Tuegels, J. Ruin estimates under interest force. Insurance: Mathematics and Economics, 16, (1995): 7 - 22.

16. Sundt, S., and Tuegels, J. The adjustment function in ruin estimates under interest force.Insurance: Mathematics and Economics, 19, (1997): 85-94.

17. 17. Thampi,K.K., Jacob, M.J., Raju, N. Ruin Probabilities under Generalized Exponential distribution. Asia-Pacific Journal of Risk and Insurance, 2(1), (2007) : 22-32.

18. Yang, H. Non-exponential bounds for ruin probabilities under interest force. Scandinavian Actuarial Journal, 1, (1999): $66-79$. 